\title{
Contractions of group representations via geometric quantization
}

\author{
Rauan Akylzhanov ${ }^{1,2}\left[\right.$ (1) Alexis Arnaudon ${ }^{1}$ \\ Received: 9 January 2019 / Revised: 25 July 2019 / Accepted: 4 September 2019 / \\ Published online: 21 November 2019 \\ (c) The Author(s) 2019
}

\begin{abstract}
We propose a general framework to contract unitary dual of Lie groups via holomorphic quantization of their coadjoint orbits, using geometric quantization. The sufficient condition for the contractibility of a representation is expressed via cocycles on coadjoint orbits. This condition is verified explicitly for the contraction of $\mathrm{SU}_{2}$ into $\mathbb{H}$. We construct two types of contractions that can be implemented on every matrix Lie group with diagonal contraction matrix.
\end{abstract}

Keywords Geometric quantization · Group contractions · Group representation

Mathematics Subject Classification Primary 22D10; Secondary 53D50

\section{Introduction}

Physicists study certain degenerations of group laws which are called contractions. In this paper, we study Lie group contractions in the context of geometric quantization. The degeneration is understood in the sense that group law becomes "more abelian", i.e. more structure constants of Lie algebra become zero. The pioneering work of Inönü and Wigner [14] is a classical original source. Their motivation was simply to relate two different physics: Galilean and Lorentzian. Contractions of representation of Lie groups and Lie algebras and their unitary duals have attracted the attention of

Rauan Akylzhanov

akylzhanov.r@gmail.com

Alexis Arnaudon

alexis.arnaudon@imperial.ac.uk

1 Department of Mathematics, Imperial College London, 180 Queen's Gate, London SW7 2AZ, $\mathrm{UK}$

2 School of Mathematical Sciences, Queen Mary University of London, Mile End Road, London E1 4NS, UK 
many authors $[5,7,12,13,19,22,23]$ and continue to be a field of active investigations; see, for example, $[3,4,9,10]$.

The first general global definition of Lie group contraction has been given in [19] where the authors study the contraction of the principal continuous series of the de Sitter groups $\mathrm{SO}(n, 1)$ to positive mass representations of both the Euclidean group $E(n)$ and the Poincare group $E_{0}(n-1,1)$. In [22], a contraction of SU(2) to the Heisenberg group $\mathbb{H}$ was studied in detail. The authors showed that the group action on the manifold depends smoothly on the contraction parameter $\varepsilon>0$. They also established transference results linking Fourier analysis on these two groups. Applying [22], Ricci and Rubin proved in [23] an analogue of de Leeuw's theorem relating $L^{p}$ norms of Fourier multipliers on $S U(2)$ and $\mathbb{H}$. A short and elegant proof of the main result of [22] was given in [5] employing Berezin quantization. Furthermore, it was shown by [8] that every semisimple Lie group can be contracted to its Cartan motion group.

Generally, one cannot expect that there always exists a contraction $\Phi_{\varepsilon}: G_{0} \rightarrow G_{1}$ for any Lie group $G_{0}$ and $G_{1}$. Indeed, let $\mathfrak{g}_{1}$ and $\mathfrak{g}_{0}$ be the Lie algebras of $G_{1}$ and $G_{0}$ and $U_{\varepsilon}: \mathfrak{g}_{1} \rightarrow \mathfrak{g}_{0}$ be differential of $\Phi_{\varepsilon}$. Then, the existence of contraction $U_{\varepsilon}$ is equivalent to the fact that $\mathfrak{g}_{0}$ is the limit in the Zariski topology on the affine variety of Lie algebras. It can be seen that not every two points in the affine variety of Lie algebras of dimension less than 6 are path-connected in the Zariski topology [18, Theorem 1]. In other words, the existence of Lie algebra contraction $U_{\varepsilon}: \mathfrak{g}_{0} \rightarrow \mathfrak{g}_{1}$ is equivalent to saying that $\mathfrak{g}_{0}$ is the limit of Lie algebras $U_{\varepsilon} \mathfrak{g}_{1}$ in the Zariski topology. Hence, we shall assume that there exists a Lie algebra contraction $U_{\varepsilon}$ of $\mathfrak{g}_{1}$ into $\mathfrak{g}_{0}$.

The aim of the paper is to show that the existence of Lie group contraction combined with the geometric quantization yields a systematic approach to link harmonic analysis on two groups. The novelty of the paper is that we do not impose any restriction on the group nor on the contraction. In addition, we tried to answer the following question.

Question 1.1 Let $G_{0}$ and $G_{1}$ be two Lie groups and let $\Phi_{\varepsilon}: G_{1} \rightarrow G_{0}$ be a Lie group contraction. Does there exist a contraction $\widehat{\Phi}_{\varepsilon}$ of $\widehat{G}_{1}$ into $\widehat{G}_{0}$ ?. In other words, does the following diagram commute

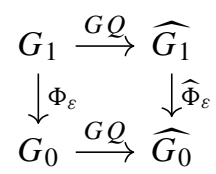

where $G Q$ stands for the geometric quantization. We shall give meaning to $\widehat{\Phi}_{\varepsilon}$ as an $M N$-contraction of $\widehat{G_{1}}$ into $\widehat{G_{0}}$.

A similar problem has been treated in [4] in the context of the algebraic HarishChandra modules. 


\section{Geometric quantization}

\subsection{General theory}

We first recall a few basic elements of the geometric quantization needed here. The procedure of quantization consists of assigning to a smooth function on a symplectic manifold $(M, \omega)$, an operator on a Hilbert space $\mathcal{H}$ with the following three properties. For each $f: M \rightarrow \mathbb{R}$, there should be an operator $\widehat{f}$ acting on $\mathcal{H}$ such that

(1) the map $f \mapsto \widehat{f}$ is linear;

(2) if $f$ is constant, $\widehat{f}$ is a multiplication operator

(3) if $\left[f_{1}, f_{2}\right]=f_{3}$, then $\left[\widehat{f_{1}}, \widehat{f_{2}}\right]=-i \hbar \widehat{f_{3}}$,

where $\hbar$ is the Planck constant.

The main ingredient in the program of geometric quantization for a symplectic manifold $(M, \omega)$ of dimension $2 n$ with symplectic (exact) 2 -form $\omega$ is to construct a Hermitian line bundle $\pi: B \rightarrow M$ with Hermitian connection $\nabla$ for which the curvature 2-form is equal to $\hbar^{-1} \omega$. Locally, the connection $\nabla$, or covariant derivative $\nabla_{X}$ for a vector field $X$ on $M$, is given by

$$
\nabla_{X}(f)=X(f)-\frac{i}{\hbar} \theta(X) f
$$

where $f$ is a function on $M$ where the connection 1-form $\theta$ can be shown to be locally related [11, Proposition 23.5] to $\omega$ as $\mathrm{d} \theta=\omega$. It is possible to construct such a line bundle on $(M, \omega)$ only if the symplectic manifold satisfies the integrality condition or is quantizable. Such a result is, for example, proved in [11, Theorem 23.9, p.489] where the integrality condition is

$$
\frac{1}{2 \pi \hbar} \int_{\Sigma} \omega \in \mathbb{Z},
$$

where $\Sigma$ is an arbitrary closed 2-surface in $M$. This condition is purely geometric and involves the computation of the holonomy of the line bundle for closed loop, which when contracted must be equal to 1 . The condition (2.2) is obtained after using Stokes's theorem.

This line bundle has a natural Hilbert space $\mathcal{H}$ consisting of square-integrable global sections with respect to the natural Liouville measure of $M$ and a Hermitian structure on the line bundle $B$. It is an inner product in the fibres denoted by $(\cdot, \cdot)$ with the property to be smooth in the sense that for a smooth section $v: M \rightarrow B,(v, v)$ is a smooth function of $M$. We now have the Hilbert space and we need to find the map $f \mapsto \widehat{f}$ satisfying the three conditions. It is given, for a section $s: M \rightarrow B$, by

$$
\widehat{f}(s)=-i \hbar \nabla_{X_{f}} s+f s
$$

where $X_{f}$ is the Hamiltonian vector field associated with the function $f$, i.e. $\omega\left(X_{f}, Y\right)=\mathrm{d} f(Y)$ for any vector field $Y$, and $f s$ is the multiplication operation. 
This operator obviously satisfies (1) and (2); the third condition is satisfied by using the properties of the covariant derivative $\nabla_{X_{f}}$; see $[11,28]$ for more details.

We will now follow [28] to expose a dynamical interpretation of $\widehat{f}$, which will give us a useful formula for later. Let $V_{f}$ be the vector field on $B$ given in a local trivialization by

$$
V_{f}=X_{f}+\hbar^{-1} L \frac{\partial}{\partial \phi}
$$

where $z=r e^{i \phi}$ is the coordinate on the fibre of $L$ and

$$
L=\theta\left(X_{f}\right)-f
$$

is the Lagrangian associated with the function $f$, or equivalently the Legendre transform of the Hamiltonian $f$. This vector field is constructed so that it is invariant under gauge transformations and $f \mapsto V_{f}$ is a Lie algebra isomorphism from $C^{\infty}(M)$ to a subalgebra of vector fields on $B$. Let $\xi_{t}$ denote the flow of $V_{f}$ which projects to $\rho_{t}$, the flow of $X_{f}$. From these two flows, we can define the action of the flow $\rho_{t}$ on the sections $s: M \rightarrow B$ by mapping it to $\widehat{\rho}_{t}$ such that

$$
\xi_{t}\left(\widehat{\rho}_{t} s(m)\right)=s\left(\rho_{t} m\right)
$$

This formula shows that $\widehat{\rho}_{t}$ is the correct pull-back of the sections such that the flow $\xi_{t}$ corresponds to only moving the base point with $\rho_{t}$. In a local trivialization, the sections $s$ and $\widehat{\rho}_{t} s$ are represented by the complex functions $\psi$ and $\widehat{\rho}_{t} \psi$ with the following relation

$$
\widehat{\rho}_{t} \psi(m)=\psi\left(\rho_{t} m\right) \exp \left(-\frac{i}{\hbar} \int_{0}^{t} L\left(\rho_{t^{\prime}} m\right) \mathrm{d} t^{\prime}\right) .
$$

It might happen that the quantum Hilbert space is reducible. A notion of polarization imposes further restrictions.

Definition 2.1 A polarization $\mathcal{P}$ of a symplectic manifold $M$ is a choice at each point $z \in M$ of a Lagrangian subspace $P(z) \subset T_{z}^{\mathbb{C}} M$ (dimension is $n$ ) satisfying the following conditions

(1) If two complex vector fields $X$ and $Y$ lie in $P(z)$ at each point $z$, then the commutator $[X, Y] \in P(z)$.

(2) The dimension of $P(z) \cap \overline{P(z)}$ is constant.

Definition 2.2 (The quantum Hilbert space). Let $\mathcal{P}$ be a polarization of $M$. A smooth section $s$ of $L \rightarrow M$ is said to be polarized if

$$
\nabla_{X} s=0, \quad \forall X \in \bar{P}
$$


The quantum Hilbert space $\mathcal{H}_{\mathcal{P}}$ associated with $\mathcal{P}$ is the closure in the prequantum Hilbert space of the space of smooth, square-integrable, polarized sections $s: L \rightarrow M$.

Definition 2.3 A polarization $\mathcal{P}$ of a symplectic manifold $M$ is called purely complex if

$$
P(z) \cap \overline{P(z)}=\varnothing
$$

for every $z \in M$.

A purely complex polarization $\mathcal{P}$ yields existence of a unique integrable almost complex structure $J$ on $M$ such that $P(z)$ is (1,0)-tangent space of $M$ for all $z \in M$.

Definition 2.4 Let $\mathcal{P}$ be a purely complex polarization of $M$ and let $J$ be the integrable almost complex structure associated with $\mathcal{P}$ such that $J(z)=i \operatorname{Id}$ on $P(z)$ and $J(z)=$ $-i$ Id on $\overline{P(z)}$. We say that $\mathcal{P}$ is a Kähler polarization if the bilinear form

$$
g(X, Y):=\omega(X, J(z) Y), \quad X, Y \in T_{z} M,
$$

is positive definite for each $z \in M$.

For a thorough exposition of the geometric quantization, we refer $[11,15,16,28]$.

\subsection{Lie group representation}

We now specify this construction to Lie group representations. Let $G$ be a locally compact Lie group with Lie algebra $\mathfrak{g}$. Each $\xi \in \mathfrak{g}$ generates a right-invariant vector field $R_{\xi}$. Let $\mu \in \mathfrak{g}^{*}$ and $\mathcal{O}_{\mu}$ be the coadjoint orbit through $\mu$. A coadjoint orbit is a symplectic manifold with the symplectic form

$$
\omega_{\mu}(\xi, \eta)=\mu([\xi, \eta]), \quad \forall \xi, \eta \in \mathfrak{g},
$$

corresponding to the reduction of the canonical symplectic form on $T^{*} G$ using the momentum map $J: T^{*} G \rightarrow \mathfrak{g}^{*}$. This form is exact and thus $\omega_{\mu}=\mathrm{d} \theta_{\mu}$, where

$$
\theta_{\mu}(\xi)=\mu(\xi) .
$$

$\left(\mathcal{O}_{\mu}, \mathrm{d} \theta_{\mu}\right)$ is a presymplectic manifold, the symplectic manifold of right cosets $G / G_{\mu}$, where $G_{\mu}$ is the stabilizer of $\mu$, i.e.

$$
G_{\mu}:=\left\{g \in G: \operatorname{Ad}_{g}^{*} \mu=\mu\right\},
$$

and its Lie algebra

$$
\mathfrak{g}_{\mu}=\left\{\xi \in \mathfrak{g}: \operatorname{ad}_{\xi}^{*} \mu=0\right\} .
$$


A coadjoint orbit $\mathcal{O}_{\mu}$ is integral if the symplectic manifold $\left(\mathcal{O}_{\mu}, \omega_{\mu}\right)$ is integral in the sense of (2.2). Let $\mathcal{O}_{\mu}$ be an integral coadjoint orbit, then there exists [11, Theorem 23.9, p.489] a holomorphic line bundle $B$ over $\mathcal{O}_{\mu}$. From (2.10) the Lagrangian $L_{\mu}$ associated with $\mu$ is given by $L_{\mu}(\xi)=\frac{1}{2} \mu(\xi)$.

Let $\pi \in \widehat{G}$ and let $\left(U_{\alpha}, \varphi_{\alpha}\right)$ be a local trivialization of $\mathcal{O}_{\mu}$. Then, it follows from (2.7) that the flow $\rho_{t}$ acts locally on the space of global holomorphic sections of $B \rightarrow \mathcal{O}_{\mu}$ via the formula

$$
\pi(g) s(m)=s\left(\operatorname{Ad}_{g^{-1}}^{*} m\right) e^{\frac{i}{\hbar} \mu \int_{m}^{m_{1}} L_{\mu} \mathrm{d} s}, m \in \mathcal{O}_{\mu}
$$

where $m_{1}=\operatorname{Ad}_{g^{-1}}^{*} m$. We used the fact that the Lagrangian $L_{\mu}$ is invariant under the coadjoint flow and the flow on the base is given by the coadjoint action; see [20, Chapter 13].

The second element of the construction is a choice of polarization. A remarkable result $[25,26]$ yields the existence of polarizing subalgebras for solvable Lie algebras. These subalgebras can be constructed algorithmically [21]. It is unknown to the authors whether there exist complex polarizations for general connected Lie groups. Therefore, we make the following assumption.

Assumption 2.5 Let $G$ be a locally compact Lie group such that for every coadjoint orbit $\mathcal{O}$ there exists invariant Kähler polarization $\mathcal{P} \subset T \mathcal{O}$.

\section{Contractions}

\subsection{Lie group contractions}

Before using geometric quantization to contract unitary duals, let us recall classic results on Lie group contractions.

Let $G_{1}$ and $G_{0}$ be two locally compact connected Lie groups of the same dimension.

Definition 3.1 We say that the family $\left\{\Phi_{\varepsilon}\right\}_{\varepsilon \geq 0}$ of differential maps

$$
\Phi_{\varepsilon}: G_{0} \rightarrow G_{1}
$$

mapping the identity $e_{G_{0}}$ of $G_{0}$ to the identity $e_{G_{1}}$ of $G_{1}$, defines a contraction of $G_{1}$ to $G_{0}$, if given any relatively compact open neighbourhood $V$ of $e_{G_{0}}$

(1) there is $\varepsilon_{V}>0$ such that for $\varepsilon<\varepsilon_{V}$ the map $\left.\Phi_{\varepsilon}\right|_{V}$ is a diffeomorphism;

(2) If $W$ is such that $W^{2} \subset V$ and $\varepsilon<\varepsilon_{V}$, then $\Phi_{\varepsilon}(W)^{2} \subset \Phi_{\varepsilon}(V)$;

(3) for $x, y \in V$

$$
\lim _{\varepsilon \rightarrow 0} \Phi_{\varepsilon}^{-1}\left(\Phi_{\varepsilon}(x) \Phi_{\varepsilon}(y)^{-1}\right)=x y^{-1} .
$$

The first two conditions are necessary for the limit of the third condition to be well defined. 
The Lie algebra can be contracted by the differential $U_{\varepsilon}=D_{e} \Phi_{\varepsilon}$ of $\Phi_{\varepsilon}$. That is, at the identity $e_{G_{0}}$

$$
U_{\varepsilon}:=D_{e} \Phi_{\varepsilon}: \mathfrak{g}_{0} \rightarrow \mathfrak{g}_{1}
$$

This map is then used to contract the Lie algebra $U_{\varepsilon}: \mathfrak{g}_{0} \rightarrow \mathfrak{g}_{1}$. Now, we fix a contraction $\Phi_{\varepsilon}: G_{0} \rightarrow G_{1}$ of $G_{1}$ to $G_{0}$ as in Definition 3.1.

\subsection{Contraction of the unitary dual}

Assume that there exists a contraction $\Phi_{\varepsilon}: G_{0} \rightarrow G_{1}$. Then, the differential $U_{\varepsilon}$ of $\Phi_{\varepsilon}$ allows us to deform the coadjoint orbits of $G_{1}$ into the coadjoint orbits of $G_{0}$. We express the adjoint action $\operatorname{Ad}_{0}$ of $G_{0}$ on $\mathfrak{g}_{0}$ as follows:

$$
\operatorname{Ad}_{0}\left(e^{X}\right)=\lim _{\varepsilon \rightarrow 0} U_{\varepsilon}^{-1} \circ \operatorname{Ad}\left(e^{U_{\varepsilon}(X)}\right) \circ U_{\varepsilon}, \quad X \in \mathfrak{g}_{0} .
$$

By the duality, we obtain the coadjoint action

$$
\operatorname{Ad}_{0}^{*}\left(e^{X}\right)=\lim _{\varepsilon \rightarrow 0}\left[U_{\varepsilon}\right]^{*} \circ \operatorname{Ad}^{*}\left(e^{U_{\varepsilon}(X)}\right) \circ\left[U_{\varepsilon}^{-1}\right]^{*}, \quad X \in \mathfrak{g}_{0},
$$

where

$$
U_{\varepsilon}^{*}(X)(Y)=Y\left(U_{\varepsilon}(X)\right), \quad X, Y \in \mathfrak{g}_{0}^{*} .
$$

The corresponding character of the contracted group $G_{0}$ is given by

$$
\chi_{\mu}\left(e^{X}\right)=\lim _{\varepsilon \rightarrow 0} e^{U_{\varepsilon}^{*}(f)(X)}, \quad \mu \in \mathfrak{g}_{0}^{*}, \quad X \in \mathfrak{g}_{0} .
$$

Following from example [2], the coadjoint orbits of the contracted group $G_{0}$ can be obtained via the following procedure

(1) Fix an element $\mu \in \mathfrak{g}_{0}^{*}$ and fix a coadjoint orbit $\mathcal{O}_{\mu}$.

(2) Transport the point $\mu$ to $\mathfrak{g}_{1}^{*}$ by using the map $\left(U_{\varepsilon}^{*}\right)^{-1}$.

(3) The action of $G_{1}$ on $\mu_{\varepsilon}=\left(U_{\varepsilon}^{*}\right)^{-1}(\mu)$ originates a $G_{1}$-coadjoint orbit $\tilde{\mathcal{O}}_{\varepsilon} \subset \mathfrak{g}_{1}^{*}$.

(4) The orbit $\tilde{\mathcal{O}}_{\varepsilon}$ is translated to $\mathfrak{g}_{0}^{*}$ by using the map $U_{\varepsilon}^{*}$, i.e.

$$
\mathcal{O}_{\mu}^{\varepsilon}=U_{\varepsilon}^{*}\left(\tilde{\mathcal{O}}_{\varepsilon}\right)
$$

The orbit method of Kirillov is a method in geometric representation theory which identifies the unitary representations of Lie groups with the canonical $G$-action on spaces of sections of specific line bundles over the coadjoint orbits. The general case of a Lie group is not yet fully understood. Vogan rigorously justified [27] the orbit method for reductive Lie groups. However, we shall restrict ourselves to solvable type I Lie groups to use results of Kostant [15] and Auslander [1]. 
Coadjoint orbits can be described by $\operatorname{Ad}_{G}$-invariant functions $f: \mathfrak{g}^{*} \rightarrow \mathbb{C}$. However, it is not clear whether the holomorphic line bundles over the coadjoint orbits $\mathcal{O}_{\varepsilon}$ are contracted properly. Therefore, we introduce.

Definition 3.2 Let $\left(U_{\alpha}, \varphi_{\alpha}\right)$ be a local trivialization of the line bundle $\mathcal{O}_{\mu} \rightarrow B$. We shall say that a family $\left\{\mathcal{O}_{\varepsilon}\right\}_{\varepsilon>0}$ of integral polarized coadjoint orbits $\mathcal{O}_{\varepsilon}$ is an admissible contraction of $\mathcal{O}_{\mu}$ if there is a family of local trivializations $\left(U_{\alpha}, c_{\alpha}^{\varepsilon}\right)$ of the line bundles $\mathcal{O}_{\varepsilon} \rightarrow B_{\varepsilon}$ such that

$$
\lim _{\varepsilon \rightarrow 0} c_{\alpha \beta}^{\varepsilon}=c_{\alpha \beta}, \quad z \in V
$$

uniformly on compact subsets $V \subset \mathbb{C}^{r}$, where $c_{\alpha \beta}^{\varepsilon}$ and $c_{\alpha \beta}$ are the transition functions associated with the trivializations $\left(U_{\alpha}, \varphi_{\alpha}\right)$ and $\left(U_{\alpha}, \varphi_{\alpha}^{\varepsilon}\right)$.

Definition 3.3 Let $B \rightarrow M$ be a holomorphic line bundle. We say a sequence of global sections $\left\{s_{n}: M \rightarrow B\right\}$ converges to the global section $s: M \rightarrow B$ if

$$
\lim _{n \rightarrow \infty} c_{\alpha \beta}^{n}(x) s_{n \beta}(x)=s_{\alpha}(x), \quad x \in U_{\alpha \beta},
$$

where $s_{n \beta}$ and $s_{\alpha}$ are the localization of $s_{n}$ and $s$ on $U_{\beta}$ and $U_{\alpha}$, respectively.

A rigorous notion of contraction of representations was first introduced in [19].

Definition 3.4 [19] We say that a representation $\sigma$ of $G_{0}$ in $\mathcal{H}$ is $M N$-contraction of the sequence $\left\{\pi^{n}\right\}$ of representations $\pi^{n}$ of $G_{1}$ in $\mathcal{H}^{n}$ if there exists a sequence $\varepsilon_{n}$ with limit 0 , a sequence of unitary operators $A_{n}: \mathcal{H}^{n} \rightarrow \mathcal{H}$ and a dense subspace $D$ of $\mathcal{H}$ such that

(1) For each $v \in D$ there is an integer $n(v)$ such that $n \geq n(v)$ implies $v \in A_{n}\left(\mathcal{H}^{n}\right)$;

(2) For each $v \in D$ and $g \in G_{0}$

$$
\lim _{n \rightarrow 0}\left\|A_{n} \pi^{n}\left(\Phi_{\varepsilon_{n}}(g)\right) A_{n}^{-1} v-\sigma(g) v\right\|_{\mathcal{H}}=0 .
$$

Question 3.5 [6] Let $G_{1}$ be a semisimple compact Lie group. Assume that there is a group contraction of $G_{1}$ to $G_{0}$. What unitary representations $\pi$ of $G_{0}$ are $M N$ contractions of the unitary irreducible representations of $G_{1}$ ?

In Theorem 3.6 we find sufficient condition on elements $\pi \in \widehat{G}$ to be contraction of a sequence $\left\{\pi_{\varepsilon}\right\} \subset \widehat{G_{1}}$. The condition is expressed in terms of the corresponding coadjoint orbits.

Theorem 3.6 Let $G_{1}$ be a compact Lie group and $G_{0}$ be a locally compact solvable type I Lie group. Let $\pi \in \widehat{G}_{0}$ be an infinite-dimensional irreducible unitary representation of $G_{0}$. Then, $\pi$ is an MN-contraction of the sequence of unitary irreducible representations if $\mathcal{O}_{\varepsilon}$ is an admissible contraction of $\left\{\mathcal{O}_{\pi}\right\}$.

Question 3.7 Is the converse true? In other words, let $\pi$ be an MN-contraction of the sequence of unitary representations $\pi_{\varepsilon} \in \widehat{G_{1}}$. Then, the coadjoint orbits $\mathcal{O}_{\varepsilon}$ are an admissible contraction of the coadjoint orbit $\left\{\mathcal{O}_{\pi}\right\}$. 
Sketch of the argument for Question 3.7 Thinking locally, we should have $M N$-contraction on each $U_{\alpha}$, i.e.

$$
\pi_{\varepsilon}(g) s_{\alpha}
$$

on each local section $s_{\alpha}$. Each local section gives rise to cocycles $c_{\alpha, \beta}$. Adding a bit more mathematical rigour, one should be able to conclude the statement of Question 3.7. $\mathrm{SU}_{2}$.

Prior to the proof of Theorem 3.6, we consider the classical contraction $\Phi_{\varepsilon}: \mathbb{H}^{1} \rightarrow$

Example 3.8 Let $G_{1}=S U(2)$ and $G_{0}=\mathbb{H}$. A Lie group contraction $\Phi_{\varepsilon}=$ $\exp \left(U_{\varepsilon}\right): G_{0} \rightarrow G_{1}$ is given by

$$
U_{\varepsilon}=\left(\begin{array}{ccc}
\varepsilon^{\frac{1}{2}} & 0 & 0 \\
0 & \varepsilon & 0 \\
0 & 0 & \varepsilon
\end{array}\right) .
$$

The coadjoint orbits $\left\{\mathcal{O}_{s}\right\}$ of $\mathrm{SU}_{2}$ are the two-dimensional spheres with half-integer radius $s \in \frac{1}{2} \mathbb{N}$, i.e.

$$
\mathcal{O}_{s}\left(\mathrm{SU}_{2}\right)=\left\{\left(x_{1}, x_{2}, x_{3}\right): x_{1}^{2}+x_{2}^{2}+x_{3}^{2}=s^{2}, \quad s \neq 0\right\}
$$

Using that $\mathcal{O}_{s}$ is diffeomorphic to the complex projective line $\mathbb{C P}^{1}$, we transport the coordinate atlas $U_{1}, U_{2} \subset \mathbb{C}$ on $\mathcal{O}_{s}$. There exists a holomorphic line bundle $\pi: B \rightarrow \mathcal{O}_{s}$ with the transition function

$$
c_{s}(z)=z^{2 s} \text {. }
$$

The existence follows from the fact that $\mathcal{O}_{s}$ is a quantizable symplectic manifold. The choice (3.14) picks up a specific bundle.

A global holomorphic section $s$ of the bundle $\pi: B \rightarrow \mathcal{O}_{s}$ is given by two holomorphic functions $s_{1}: U_{1} \rightarrow \mathbb{C}$ and $s_{2}: U_{2} \rightarrow \mathbb{C}$ related by

$$
s_{1}(z)=c_{s}(z) s_{2}\left(\frac{1}{z}\right), \quad z \in U_{1} \cap U_{2} .
$$

Expanding holomorphic functions $s_{1}$ and $s_{2}$ into Taylor series

$$
\sum_{n=0} a_{m} z^{m}=z^{2 s} \sum_{n=0} \tilde{a}_{n} z^{-n} a
$$

in respective coordinates and equating coefficients, we find that $\tilde{a}_{n}=a_{n}=0$ for $m>2 s$ and $\tilde{a}_{0}=a_{2 s}, \tilde{a}_{1}=a_{2 s-1}$, etc. Hence, the section $s$ is given by a polynomial

$$
s(z)=\sum_{n=0}^{2 s} a_{n} z^{n} .
$$


The coadjoint orbits $\mathcal{O}_{\lambda}$ of the Heisenberg group $\mathbb{H}$ are given by

$$
\mathcal{O}_{\lambda}=\left\{z \in \mathbb{C}: \frac{z+z^{*}}{2}=\lambda, \quad \frac{z-z^{*}}{2}=0\right\}
$$

In other words, the orbits $\mathcal{O}_{\lambda}$ are planes through the point $\mathcal{O}_{\lambda}=\{(\lambda, 0)\}$. There exists a holomorphic line bundle $\pi: B \rightarrow \mathcal{O}_{\lambda}$ with the transition function

$$
c^{\lambda}(z)=e^{i \lambda z}, z \in \mathbb{C} .
$$

A global holomorphic section $F$ of $\pi: B \rightarrow \mathcal{O}_{\lambda}$ is a holomorphic function. We deform the transition function $c_{S}$ defined in (3.14) as follows:

$$
c_{s}^{\varepsilon}(z)=(1+i \varepsilon z)^{2 s_{\varepsilon}},
$$

where

$$
s_{\varepsilon}=\left[\begin{array}{l}
\lambda \\
\varepsilon
\end{array}\right], \quad \lambda \in \mathbb{R} .
$$

An elementary calculation then yields

$$
\lim _{\varepsilon \rightarrow 0}(1+i \varepsilon z)^{2 s_{\varepsilon}}=e^{i \lambda z}
$$

This shows the condition (3.9) holds true.

The extension of Example 3.8 to a broader class of Lie groups (e.g. semisimple Lie groups) remains a challenging problem [24] and will be considered elsewhere.

Proof of Theorem 3.6 For every $g \in G_{0}$ the sections $\pi^{\varepsilon}\left(g^{\varepsilon}\right) s^{\varepsilon}$ converge to the section $\pi(g) s_{\alpha}$ in the sense of Definition 3.3, i.e.

$$
\lim _{\varepsilon \rightarrow 0} \pi^{\varepsilon}\left(g^{\varepsilon}\right) s_{\alpha}^{\varepsilon}=\pi(g) s_{\alpha},
$$

uniformly for every compact subset $V \subset U_{\alpha} \cap U_{\beta}$.

Let $\pi$ be a fixed unitary irreducible representation, and let us denote by $\mathcal{O}_{\pi} \subset$ $\mathfrak{g}_{0}$ the coadjoint orbit associated with $\pi$. Let us denote by $\pi_{\varepsilon}$ the representations corresponding to the coadjoint orbits $\mathcal{O}_{\pi}^{\varepsilon}$. The latter is a contraction of $\mathcal{O}_{\pi}$ by the hypothesis. Let $\left(U_{\alpha}, \varphi_{\alpha}\right)$ be a local trivialization of $\mathcal{O}_{\pi} \rightarrow B$, and let us fix an arbitrary element $s \in \mathcal{H}^{\pi}$. We denote by $s_{\alpha}$ its localization to $U_{\alpha} \subset O_{\pi}$. Abusing our notation, we shall also write $s_{\alpha}$ for the coordinate representation of $s_{\alpha}$, i.e. $s_{\alpha}(z)=$ $s_{\alpha}\left(\varphi_{\alpha}^{-1}(z)\right), \quad z \in \mathbb{C}^{r}$. Let us denote

$$
\operatorname{Ad}_{g}^{* \varepsilon}: \mathcal{O}_{\pi} \rightarrow \mathcal{O}_{\pi}^{\varepsilon}
$$


given by

$$
\operatorname{Ad}_{g}^{* \varepsilon}(X)=U_{\varepsilon}^{*} \operatorname{Ad}_{g}^{* 1}\left(U_{\varepsilon}^{-1}\right)^{*}(X), \quad X \in \mathfrak{g}_{0} .
$$

Indeed, by (2.13)

$$
\pi^{\varepsilon}(g) s_{\alpha}(m)=s_{\alpha}\left(\operatorname{Ad}_{g}^{* \varepsilon} m\right) \psi_{\mu}(m, g)
$$

where we denote

$$
\psi_{\mu}(m, g)=\exp \left(\frac{i}{2 \hbar} \underset{\gamma: m \rightarrow \operatorname{Ad}_{g}^{*} m}{ } \mathcal{L}_{\mu} \mathrm{d} s\right)
$$

Thus, we get

$$
\begin{aligned}
\left|\pi(g) s_{\alpha}(m)-\pi^{\varepsilon}\left(\Phi_{\varepsilon}(g)\right) s_{\alpha}^{\varepsilon}(m)\right| \leq & \left|s_{\alpha}\left(\operatorname{Ad}_{g}^{*} m\right)-s_{\alpha}^{\varepsilon}\left(\operatorname{Ad}_{g}^{* \varepsilon} m\right)\right| \\
& +C\left|\psi_{\mu}(m, g)-\psi_{\mu_{\varepsilon}}\left(m, \Phi_{\varepsilon_{n}}(g)\right)\right|
\end{aligned}
$$

where we used (3.27) and the fact that $\left|\psi_{\mu}(m, g)\right| \leq 1$. This shows that

$$
\lim _{\varepsilon \rightarrow 0}\left|s_{\alpha}\left(\operatorname{Ad}_{g}^{*} m\right)-s_{\alpha}^{\varepsilon}\left(\operatorname{Ad}_{g}^{* \varepsilon} m\right)\right| \quad \text { uniformly in } U_{\alpha}
$$

The sections $s$ and $s^{\varepsilon}$ are holomorphic. Hence, their localizations $s_{\alpha}$ and $s_{\alpha}^{\varepsilon}$ admit Taylor expansions

$$
\begin{gathered}
s_{\alpha}(z)=\sum_{n \in \mathbb{N}^{r}} c_{k}^{\alpha} z^{k}, z \in \varphi\left(U_{\alpha}\right) \subset \mathbb{C}^{r}, \\
s^{\varepsilon} \alpha(z)=\sum_{n \in \mathbb{N}^{r}} c_{k}^{\alpha \varepsilon} z^{k}, z \in \varphi\left(U_{\alpha}\right) \subset \mathbb{C}^{r} .
\end{gathered}
$$

Since every coadjoint orbit $\mathcal{O}^{\pi} \varepsilon$ is compact, the Hilbert space $\mathcal{H}^{\varepsilon}$ of global holomorphic sections $s^{\varepsilon}$ is finite dimensional in view of the Riemann-Roch theorem. The dimension of the representation space is given [17] by

$$
\operatorname{dim}\left(\pi^{\varepsilon}\right)=\operatorname{vol}\left(\mathcal{O}_{\pi}^{\varepsilon}\right)
$$

Hence, we get

$$
s_{\alpha}^{\varepsilon}(z)=\sum_{|k| \leq N_{\varepsilon}} c_{k}^{\alpha \varepsilon} z^{k}, \quad z \in \mathbb{C}^{r}
$$

in where $N_{\varepsilon} \geq \operatorname{dim}\left(\pi^{\varepsilon}\right)$. Let us choose 


$$
c_{k}^{\alpha \varepsilon}=c_{k}^{\alpha}, \quad|k| \leq N_{\varepsilon} .
$$

We have

$$
\begin{aligned}
\left|s_{\alpha}\left(\operatorname{Ad}_{g}^{*} m\right)-s_{\alpha}^{\varepsilon}\left(\operatorname{Ad}_{g}^{* \varepsilon} m\right)\right| & =\left|s_{\alpha}\left(\operatorname{Ad}_{g}^{*} m\right)-s_{\alpha}^{\varepsilon}\left(\operatorname{Ad}_{g}^{*} m\right)+s_{\alpha}^{\varepsilon}\left(\operatorname{Ad}_{g}^{*} m\right)-s_{\alpha}^{\varepsilon}\left(\operatorname{Ad}_{g}^{* \varepsilon} m\right)\right| \\
& \leq\left|s_{\alpha}\left(\operatorname{Ad}_{g}^{*} m\right)-s_{\alpha}^{\varepsilon}\left(\operatorname{Ad}_{g}^{*} m\right)\right|+\left|s_{\alpha}^{\varepsilon}\left(\operatorname{Ad}_{g}^{*} m\right)-s_{\alpha}^{\varepsilon}\left(\operatorname{Ad}_{g}^{* \varepsilon} m\right)\right|
\end{aligned}
$$

Since $s_{\alpha}^{\varepsilon}$ is a continuous function and using (3.5), we get that the second term in the last inequality of (3.35) goes to zero as $\varepsilon \rightarrow 0$.

Composing (3.30) and (3.31) and (3.33), we get

$$
\left|s_{\alpha}\left(\operatorname{Ad}_{g}^{*} m\right)-s_{\alpha}^{\varepsilon}\left(\operatorname{Ad}_{g}^{*} m\right)\right|=\sum_{k \in \mathbb{N}^{r}:|k| \geq N_{\varepsilon}} c_{k}^{\alpha}\left(\operatorname{Ad}_{g}^{*} m\right)^{k} .
$$

The last sum converges to zero uniformly by the holomorphicity of $s_{\alpha}$. This establishes (3.29). By the existence of contraction, we immediately obtain that

$$
\lim _{\varepsilon \rightarrow 0} \int_{\gamma: m \rightarrow \operatorname{Ad}_{g}^{*} m} \mathcal{L}_{\mu} \mathrm{d} s=\int_{\gamma_{\varepsilon}: m \rightarrow \operatorname{Ad}_{\Phi_{\varepsilon}(g)}^{* \varepsilon} m} \mathcal{L}_{\mu}^{\varepsilon} \mathrm{d} s
$$

Thus, we have just shown (3.23).

This completes the proof.

\section{Two classes of contractions}

We exhibit here two types of contractions that can be implemented on any matrix Lie group and which encompass most of the contractions with a diagonal contraction matrix $U_{\varepsilon}$. First note that in the limit $\varepsilon \rightarrow 0$, the important terms in the matrix $U_{\varepsilon}$ will be the lowest powers in $\varepsilon$. Notice also that $\varepsilon$ is arbitrary, so only the relative scaling between terms is important. From these considerations, we will study the following two forms of the contraction matrices $U_{\varepsilon}$.

$$
\begin{aligned}
U_{\mathrm{IW}} & =\varepsilon \mathrm{Id}+(1-\varepsilon) u \\
U_{\text {anti-IW }} & =\varepsilon^{2} \mathrm{Id}+\varepsilon(1-\varepsilon) u,
\end{aligned}
$$

where $u$ is a matrix to be determined and does not depend on $\varepsilon$. The first contraction is the classical Inönü-Wigner contraction, and the second will be called the antiInönü-Wigner contraction for reasons that will become obvious soon. First, we need to define a particular decomposition of the Lie algebra.

Definition 4.1 For a diagonal matrix $u$ of dimension $n=\operatorname{dim}\left(\mathfrak{g}_{1}\right)$, define the two subspaces of $V=\mathbb{R}^{n}$, the underlying vector space of $\mathfrak{g}_{1}$ by 


$$
u V_{N}=0 \quad \text { and } \quad u V=V_{R}
$$

Furthermore, these subspaces satisfy

$$
\mathfrak{g}_{1}=V_{N} \oplus V_{R}
$$

We now want to derive a geometrical condition on $u$ that will ensure that we obtain a contraction. For this, we will need the following object.

Definition 4.2 (IW-tensor). We the following 2-tensor

$$
T[u](\xi, \eta):=u^{2}[\xi, \eta]-u([u \xi, \eta]+[\xi, u \eta])+[u \xi, u \eta]
$$

that we will call the IW-tensor.

In the case when $T[u](\xi, \eta)=0$, it reduces to the well-known Nijenhuis tensor of complex geometry.

We introduce the following conditions on $u$ in terms of this tensor and the subspaces defined above.

Theorem 4.3 The following conditions on u give a valid Lie algebra contraction for a choice of splitting $\mathfrak{g}_{1}=V_{N} \oplus V_{R}$ if

(1) $T[u](\xi, \eta) \in V_{R}, U_{\text {IW }}$ is an Inönü-Wigner contraction, or

(2) $T[u](\xi, \eta) \in V_{N}, U_{\text {anti-IW }}$ is an anti-Inönü-Wigner contraction.

Proof We begin by proving the IW contraction condition by rewriting

$$
U_{\mathrm{IW}}=(1-\varepsilon)(\lambda \mathrm{Id}+u),
$$

where $\lambda=\frac{\varepsilon}{1-\varepsilon}$ converges to 0 when $\varepsilon \rightarrow 0$. We first rewrite the Lie bracket using (4.2) to get

$$
U^{-1}[U \xi, U \eta]=U^{-1}\left\{\lambda^{2}[\xi, \eta]+\lambda([u \xi, \eta]+[\xi, u \eta])+[u \xi, u \eta]\right\}
$$

We then have to ensure that the inner term is in $V_{R}$, where $U$ is invertible in the limit $\varepsilon \rightarrow 0$. First notice that from the definition of the contraction (4.2) we have the relation $U^{-1}(\lambda \zeta)=\zeta-U^{-1} u \zeta$ for an arbitrary element $\zeta \in \mathfrak{g}$. We can thus rewrite the previous equation as

$$
\begin{aligned}
U^{-1}[U \xi, U \eta]= & \lambda[\xi, \eta]+[u \xi, \eta]+[\xi, u \eta] \\
& +U^{-1}\{-\lambda u[\xi, \eta]-u([u \xi, \eta]+[\xi, u \eta])+[u \xi, u \eta]\} \\
= & \lambda[\xi, \eta]+[u \xi, \eta]+[\xi, u \eta]-u[\xi, \eta] \\
& +U^{-1}\left\{u^{2}[\xi, \eta]-u([u \xi, \eta]+[\xi, u \eta])+[u \xi, u \eta]\right\} .
\end{aligned}
$$


This computation assumes that $u$ is diagonal, and will not apply for more general contractions. Then, as $U^{-1}$ is only defined on $V_{R}$ in the limit $\varepsilon \rightarrow 0$, to the condition for $u$ to be a valid contraction, i.e. for the limit $\varepsilon \rightarrow 0$ to exist translates to the condition on the IW-tensor,

$$
T[u](\xi, \eta) \in V_{R} .
$$

The new Lie bracket is

$$
[\xi, \eta]_{0}=[\xi, \eta]^{\prime}+u^{-1} T[u](\xi, \eta)
$$

where we defined

$$
\begin{aligned}
{[\xi, \eta]^{\prime}: } & =[u \xi, \eta]+[\xi, u \eta]-u[\xi, \eta] \\
T[u](\eta, \xi) & =[u \xi, u \eta]-u[\xi, \eta]^{\prime} .
\end{aligned}
$$

We now turn to the condition for the anti-IW contraction where we can still use the equivalent formulation

$$
U_{\mathrm{anti}-\mathrm{IW}}=\varepsilon(\lambda I d+u)
$$

where $\lambda=\frac{\varepsilon}{1-\varepsilon}$. We then follow the same procedure as before. We first expand the bracket using (4.11) to get

$$
U^{-1}[U \xi, U \eta]=U^{-1}\left\{\varepsilon^{2 \alpha}\left(\lambda^{2}[\xi, \eta]+\lambda([u \xi, \eta]+[\xi, u \eta])+[u \xi, u \eta]\right)\right\}
$$

We now have the relation $U^{-1}(\varepsilon \lambda \zeta)=\zeta-U^{-1}(\varepsilon u \zeta)$, so we can rewrite

$$
\begin{aligned}
U^{-1}[U \xi, U \eta]= & \varepsilon(\lambda[\xi, \eta]+[u \xi, \eta]+[\xi, u \eta]) \\
& +U^{-1}\left\{\varepsilon^{2}(-\lambda u[\xi, \eta]-u([u \xi, \eta]+[\xi, u \eta])+[u \xi, u \eta])\right\} \\
= & \varepsilon(\lambda[\xi, \eta]+[u \xi, \eta]+[\xi, u \eta]-\varepsilon u[\xi, \eta]) \\
& +U^{-1}\left\{\varepsilon^{2}\left(u^{2}[\xi, \eta]-u([u \xi, \eta]+[\xi, u \eta])+[u \xi, u \eta]\right)\right\} .
\end{aligned}
$$

We can go further in the computation to extract non-vanishing terms by using the relation $U^{-1}\left(\varepsilon \lambda\left(\varepsilon \lambda^{-1} \zeta\right)\right)=\varepsilon \lambda^{-1} \zeta-U^{-1}\left(\varepsilon u \varepsilon \lambda^{-1} \zeta\right)$ to get

$$
\begin{aligned}
U^{-1}[U \xi, U \eta]= & \varepsilon(\lambda[\xi, \eta]+[u \xi, \eta]+[\xi, u \eta]-\varepsilon u[\xi, \eta]) \\
& +\varepsilon \lambda^{-1}\left(u^{2}[\xi, \eta]-u([u \xi, \eta]+[\xi, u \eta])+[u \xi, u \eta]\right) \\
& +U^{-1}\left\{\varepsilon^{2} \lambda^{-1} u\left(u^{2}[\xi, \eta]-u([u \xi, \eta]+[\xi, u \eta])+[u \xi, u \eta]\right)\right\}
\end{aligned}
$$


Note that $\lambda \propto \varepsilon$ when $\varepsilon \rightarrow 0$, thus the limit $\varepsilon \rightarrow 0$ in the previous equation gives the new Lie bracket only in terms of the IW-tensor

$$
[\xi, \eta]_{0}=T[u](\xi, \eta)
$$

In this case, the condition for existence of the contraction is given by

$$
T[u](\xi, \eta) \in V_{N}
$$

We can make a couple of interesting remarks at this point.

(1) If $T[u](\xi, \eta)=0, \forall \xi, \eta \in \mathfrak{g}$, then the contracted Lie algebra is isomorphic to the original Lie algebra and $u$ is a Nijenhuis tensor. This can be seen by the fact that $T[u](\xi, \eta)=0$ is equivalent to $[u \xi, u \eta]=u[\xi, \eta]^{\prime}$, and thus, $u$ defines a homomorphism of Lie algebras between $[\cdot, \cdot]$ and $[\cdot, \cdot]_{0}=[\cdot, \cdot]^{\prime}$.

(2) If $[\xi, \eta]^{\prime}=0, \forall \xi, \eta \in \mathfrak{g}, u$ is a derivation of the Lie algebra $\mathfrak{g}$ as it will satisfy the Leibniz rule $u[\xi, \eta]=[u \xi, \eta]+[\xi, u \eta]$. The new bracket is then simply $[\xi, \eta]_{0}=u^{-1}[u \xi, u \eta]$, but this would also mean that $u$ is invertible everywhere, and thus, the two Lie algebras would be isomorphic.

From these two remarks we get that in order to have a contraction, $u$ must not be a homomorphism of Lie algebras nor a derivation of Lie algebras.

We can even go further and give an extra condition on the splitting, as well the type of Lie algebra that results from the contraction.

Theorem 4.4 Given a finite-dimensional complex Lie algebra $\mathfrak{g}_{1}$, for any subspace $\mathfrak{u}$ of a subalgebra of $\mathfrak{g}_{1}$, let $V_{R}=\mathfrak{u}$ yield an IWS contraction to a semi-direct algebra and let $V_{N}=\mathfrak{u}$ yield an anti-IWS contraction to a nilpotent Lie algebra.

Proof Let us first look at the anti-IWS contractions. Let $V_{N} \subset \mathfrak{p}$, so that we have $\left[V_{N}, V_{N}\right] \subset V_{N}$ and $V_{N}$ are nilpotent. One can easily check that $T[u]\left(\xi_{N}, \eta_{N}\right)=0$, and that

$$
T[u]\left(\xi_{R}, \eta_{R}\right)=-\left[\xi_{R}, \eta_{R}\right]_{R}+\left[\xi_{R}, \eta_{R}\right]=\left[\xi_{R}, \eta_{R}\right]_{N} \subset V_{N}
$$

The last commutation trivially gives $T[u]\left(\xi_{N}, \eta_{R}\right)=\left[\xi_{N}, \eta_{R}\right]_{R}-\left[\xi_{N}, \eta_{R}\right]_{R}=0$. We thus have that if $V_{N} \subset \mathfrak{b}$, then the contraction is anti-IWS.

We now look at the IWS contractions. We first have $T[u]\left(\xi_{R}, \eta_{R}\right)=0$ and then $T[u]\left(\xi_{R}, \eta_{R}\right)=\left[\xi_{R}, \eta_{R}\right]_{R} \subset V_{R}$ and $T[u]\left(\xi_{N}, \eta_{R}\right)=0$. This gives the corresponding result.

Acknowledgements The authors would like to thank Anthony Dooley and Fulvio Ricci along with Michael Ruzhansky for their support and advice while this research was being done. Alexis Arnaudon is supported by the EPSRC through award EP/N014529/1 funding the EPSRC Centre for Mathematics of Precision Healthcare. Rauan Akylzhanov was partially supported by the EPSRC Grant EP/R003025/1. 
Open Access This article is distributed under the terms of the Creative Commons Attribution 4.0 International License (http://creativecommons.org/licenses/by/4.0/), which permits unrestricted use, distribution, and reproduction in any medium, provided you give appropriate credit to the original author(s) and the source, provide a link to the Creative Commons license, and indicate if changes were made.

\section{References}

1. Auslander, L., Kostant, B.: Quantization and representations of solvable Lie groups. Bull. Am. Math. Soc. 73, 692-695 (1967)

2. Arratia, O., Del Olmo, M.A.: Contraction of representations of $1+1$ kinematical groups and quantization. Int. J. Modern Phys. A 12(1), 125-130 (1997). IWCQIS 96 (Dubna, 1996)

3. Bernstein, J., Higson, N., Subag, E.: Algebraic families of Harish-Chandra Pairs. (2016). arXiv: 1610.03435

4. Bernstein, J., Higson, N., Subag, E.: Contractions of representations and algebraic families of HarishChandra modules (2017). arXiv:1703.04028

5. Cahen, B.: Contraction de SU(2) vers le groupe de Heisenberg et calcul de Berezin. Beiträge Algebra Geom. 44(2), 581-603 (2003)

6. Cahen, B.: Some remarks on the notion of contraction of lie group representations. Math. Morav. 14(1), 35-46 (2010)

7. Dooley, A.H.: Contractions of Lie groups and applications to analysis. In: Topics in Modern Harmonic Analysis, vol. I, II (Turin/Milan, 1982), pp. 483-515. Ist. Naz. Alta Mat. Francesco Severi, Rome (1983)

8. Dooley, A.H., Rice, J.W.: On contractions of semisimple lie groups. Trans. Am. Math. Soc. 289, 185-202 (1985)

9. Fialowski, A., Penkava, M.: Formal deformations, contractions and moduli spaces of Lie algebras. Int. J. Theoret. Phys. 47(2), 561-582 (2008)

10. Gromov, N.: From Wigner-Inon̈ü group contraction to contractions of algebraic structures. Acta Phys. Hung. A Heavy Ion Phys. 19(3), 209-212 (2004)

11. Hall, B.C.: Quantum Theory for Mathematicians, Volume 267 of Graduate Texts in Mathematics. Springer, New York (2013)

12. Higson, N.: The Mackey analogy and $K$-theory. In Group representations, ergodic theory, and mathematical physics: a tribute to George W. Mackey, volume 449 of Contemp. Math., pp. 149-172. American Mathematical Society, Providence (2008)

13. Higson, N.: On the analogy between complex semisimple groups and their Cartan motion groups. In Noncommutative geometry and global analysis, volume 546 of Contemp. Math., pp. 137-170. American Mathematical Society, Providence (2011)

14. Inonu, E., Wigner, E.P.: On the contraction of groups and their representations. Proc. Natl. Acad. Sci. USA 39, 510-524 (1953)

15. Kostant, B.: Quantization and Unitary Representations. Lectures in Modern Analysis and Applications III, pp. 87-208. Springer, Berlin (1970)

16. Kirillov, A.A.: Geometric Quantization. Dynamical Systems IV, pp. 137-172. Springer, Berlin (1990)

17. Kirillov, A.A.: Lectures on the Orbit Method, vol. 64. American Mathematical Society, Providence (2004)

18. Kirillov, A., Neretin, Y.: The Variety of $A_{n}$ of $n$-Dimensional Lie Algebra Structures. American Mathematical Society, Providence (1987)

19. Mickelsson, J., Niederle, J.: Contractions of representations of de Sitter groups. Commun. Math. Phys. 27(3), 167-180 (1972)

20. Marsden, J.E., Ratiu, T.S.: Introduction to Mechanics and Symmetry: A Basic Exposition of Classical Mechanical Systems. Springer, Berlin (2010)

21. Oussa, V.: Computing vergne polarizing subalgebras. Linear Multilinear Algebra 63(3), 578-585 (2015)

22. Ricci, F.: A contraction of SU(2) to the Heisenberg group. Monatsh. Math. 101(3), 211-225 (1986)

23. Ricci, F., Rubin, R.L.: Transferring Fourier multipliers from SU(2) to the Heisenberg group. Am. J. Math. 108(3), 571-588 (1986)

24. Schodler, T., Valette, A.: Private communication (2017) 
25. Vergne, M.: Construction de sous-algèbres subordonnées à un élément du dual d'une algèbre de Lie résoluble. C. R. Acad. Sci. Paris Sér. A-B 270, A173-A175 (1970)

26. Vergne, M.: Construction de sous-algèbres subordonnées à un élément du dual d'une algèbre de Lie résoluble. C. R. Acad. Sci. Paris Sér. A-B 270, A704-A707 (1970)

27. Vogan, D.: The method of coadjoint orbits for real reductive groups. Represent. Theory Lie Groups (Park City, UT, 1998) 8, 179-238 (1998)

28. Woodhouse, N.: Geometric Quantization. Oxford Mathematical Monographs. Oxford University Press, Oxford (1980)

Publisher's Note Springer Nature remains neutral with regard to jurisdictional claims in published maps and institutional affiliations. 